\title{
A New Type of Wind Tunnel for the Evaluation of Curved Motion
}

\author{
James Keogh ${ }^{1}$ and Tracie Barber ${ }^{2}$ \\ UNSW Australia, Sydney, NSW, 2052, Australia \\ Sammy Diasinos ${ }^{3}$ \\ Macquarie University, Sydney, NSW, 2109, Australia \\ and \\ Graham Doig ${ }^{4}$ \\ California Polytechnic State University, San Luis Obispo, CA, 93047
}

While aerodynamic analysis of the flow conditions during cornering can be an important design parameter for applications such as automobiles, vessels, and highly maneuverable aircraft, it can be difficult to simulate. This type of motion requires controlled and repeatable flow curvature, relative to the model. A new design to allow experimental testing for this condition has been developed, and by testing in a noninertial reference frame, flow curvature is achieved in the absence of a static pressure gradient. Initial results have demonstrated the ability of the concept to produce the correct flowfield and the new design offers potential for new experimental investigations that were previously unachievable.

\section{Nomenclature}

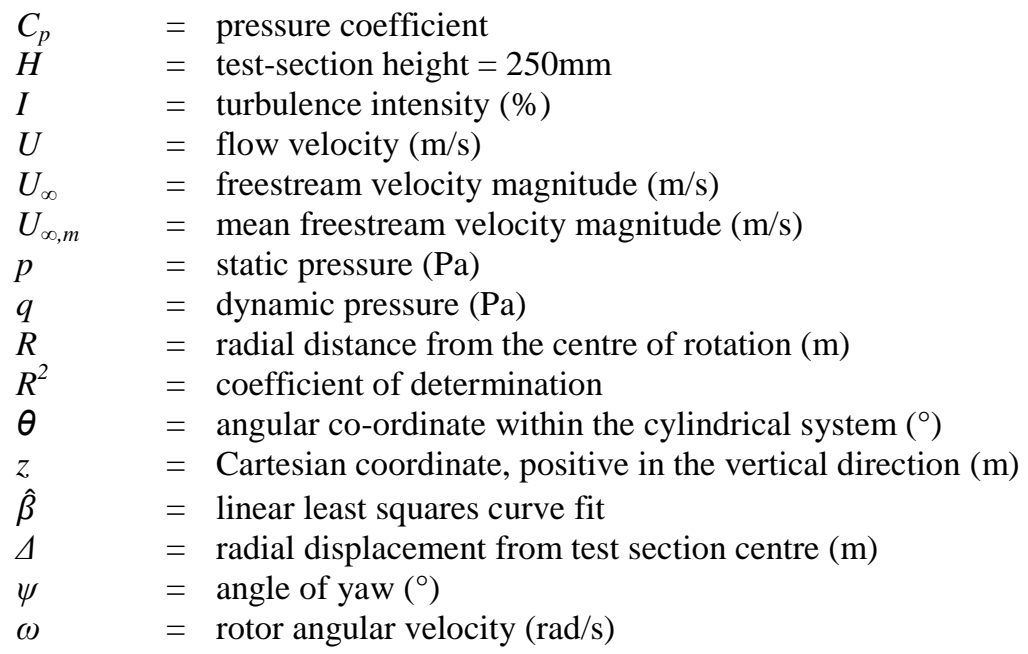

CFD $=$ computational fluid dynamics

COR $=$ center of rotation

\footnotetext{
${ }^{1} \mathrm{PhD}$ Candidate, School of Mechanical and Manufacturing Engineering, UNSW Australia, AIAA Member

${ }^{2}$ Associate Professor, School of Mechanical and Manufacturing Engineering, UNSW Australia.

${ }^{3}$ Lecturer, Science Faculty, Department of Engineering, Macquarie University.

${ }^{4}$ Assistant Professor, Aerospace Engineering Department, California Polytechnic State University; co-appointment Adjunct

Lecturer, School of Mechanical and Manufacturing Engineering, UNSW Australia; AIAA Senior Member
} 


\section{Introduction}

new experimental rig has been developed for the aerodynamic analysis of curved motion on a two-dimensional plane. The intention of the design was to provide correct and repeatable flow conditions that are representative of the true cornering condition.

For vehicles in close ground proximity there has always been difficulties in experimentally analyzing the aerodynamic performance through a corner. For some applications, such as motorsport, it is the condition where optimum aerodynamic performance is most desirable. A number of previous methods have been pursued with limited success, and none are able to sustain the correct flowfield, limiting the potential for aerodynamic development in this unique condition ${ }^{1}$.

In conventional wind tunnels dynamic rigs are commonly used to reproduce maneuvers in freestream conditions. In close ground proximity the ground's aerodynamic interaction must also be considered. This requires curved flow to be produced in the presence of a representative ground plane. This becomes near impossible for any dynamic rig, as was further discussed by Keogh et. $\mathrm{al}^{2}$.

The present concept seeks to achieve flow conditions representative of steady-state two-dimensional cornering, as is shown in Fig. 1. This ideal condition involves the flow passing along an arc of constant radius curvature relative to the model. The tangential velocity in the rotating reference frame became proportional to the angular velocity and distance from the centre of rotation. The outer side of the object will be in a region of higher tangential velocity relative to the inner side, while the static pressure remains constant.

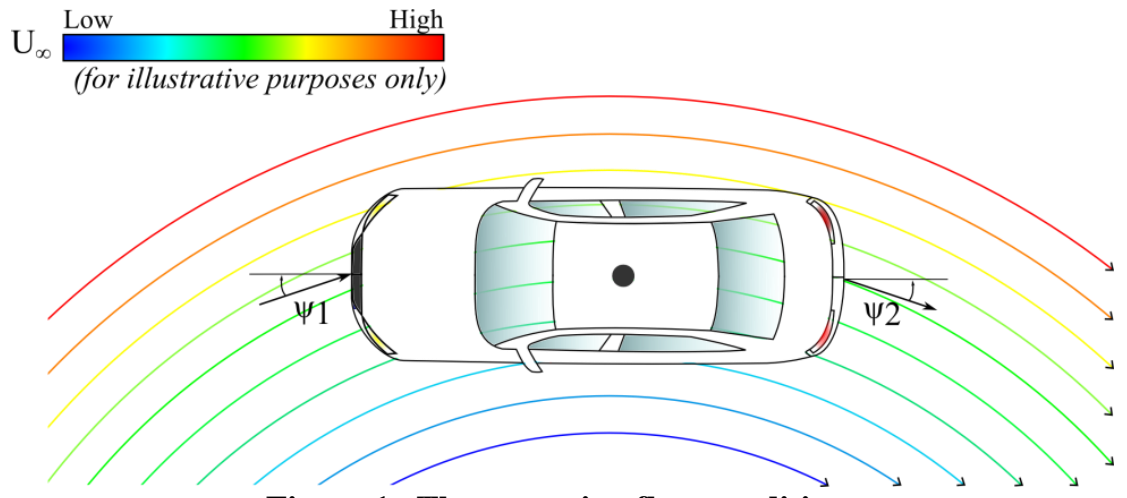

Figure 1. The cornering flow conditions

For vehicles the significant effects of this condition have been studied both numerically, and in on-road conditions ${ }^{3-}$

7. These studies have indicated that the severity of these effects is highly geometry dependent. An inverted wing in close ground proximity demonstrated sensitivity to the change in local Reynolds number ${ }^{3}$, whereas a bluff body was more sensitive to the change in flow angle from front to rear, and acceleration of the flow along the side of the body ${ }^{4}$.

The new experimental rig design is shown in Fig. 2 and uses a $336 \times 250 \mathrm{~mm}$ test section which is capable of simulating a $750 \mathrm{~mm}$ mean radius corner. To achieve this a rotating reference frame was required for the test section to allow the correct curved flow condition in the absence of a static pressure gradient. The experiment was thus constructed in two sections, the stator and the rotor. The lower section rotated about the vertical axis and was capable of a maximum angular velocity of $13.33 \mathrm{rad} / \mathrm{s}$ which was equivalent a maximum mean tangential velocity of $10 \mathrm{~m} / \mathrm{s}$.

The stator component was located centrally and consists of a bellmouth inlet to an axial fan, followed by a straight circular tunnel section. The flow passes through a sealed rotary joint into the rotor component. Turning vanes are used to turn the flow through $90^{\circ}$ into the plane of the test-section. Then a 2:1 contraction nozzle is used to deliver flow to the test-section where a model would be positioned. The extended test section then has an open exit, where the net outflow, travels radially outwards from the experimental rig.

\section{Concept}

If we regard the surrounding environment as an inertial reference frame, when any object travels in a curved path it is in a non-inertial reference frame. Considering this motion as being through a constant speed, constant radius corner, a stationary body within that rotating reference frame is undergoing constant acceleration. In order to recreate this flow environment, placing the body within a representative non-inertial reference frame is required. 
Dependent on any wind tunnel's alignment with respect to the Earth's axis of rotation, the flow would want to follow a very slight curvature, so slight it would be likely immeasurable. This occurs due to the Coriolis effect ${ }^{8}$ due to which any object in motion within a non-inertial reference frame will naturally deflect in a direction which is dependent on one's location within that reference frame. This design differs in that utilizes a far higher angular velocity to enhance this effect to create more significant relative flow curvature.
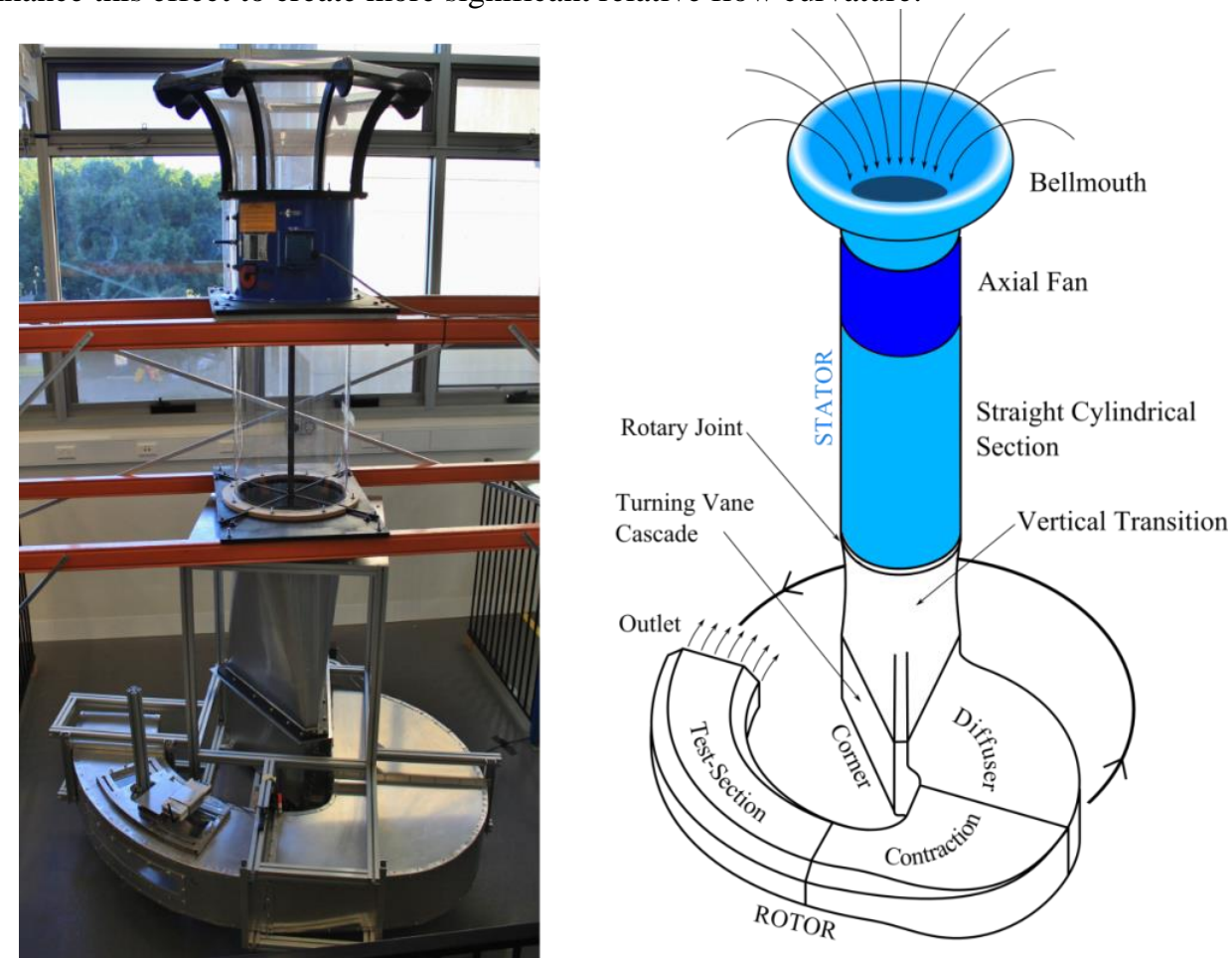

Figure 2. Experimental configuration showing primary components

To explain the basic principle behind the design it becomes simpler to regard an isolated particle. If a single fluid particle travelling at a constant velocity enters a rotating reference frame at the centre of rotation, the initial velocity and total pressure remain unchanged - despite the change in reference frame. This only occurs as the particle is entering at the exact centre. As the displacement of the particle from the centre increases, its velocity, and relative total pressure (within the rotating reference frame) will increase. If left unimpeded the particle will assume a spiraling outwards path due to its Coriolis acceleration within this frame. An anti-clockwise rotation causes the particle to deflect to the right as shown in Fig. 3. Noting the equal time increments labeled 1 to 5, it can be observed that the distance travelled in each interval will increase as the particle travels further from the centre. This is due to the addition of the $\omega \mathrm{R}$ component within the rotating reference frame, while the initial velocity, $\mathrm{u}_{0}$, remains constant. In the absolute reference frame the particle is simply following its normal straight path it is only due to the acceleration of the reference frame it is being observed from that the change in flow conditions is observable.

If the same fluid particle was to enter the rotating reference frame with an initial velocity $\mathrm{U}$, but, while gaining velocity with radial displacement, the initial velocity component is reduced to zero. At time interval 5, shown in Fig. 4, the particle (unimpeded) would follow a constant radius arc, maintaining a constant distance from the centre of rotation. Observing the same effect in the absolute reference frame, it would show that the same particle has simply been decelerated from its initial velocity to zero at a determined location.

In the absolute reference frame this method creates stationary flow for the object to pass through, hence allowing replication of reality. The same concept of passing the model through stationary air was used before the first wind tunnel was ever constructed, through the use of the whirling $\mathrm{arm}^{9}$. The design continuously spun a model in a circular path through the air in order to create a relative velocity between the fluid and the model. However, by continually passing the model around in circles it was travelling through its own wake - creating a very turbulent flowfield with a further whirlpool-like effect. Dependent on the aerodynamic drag of the equipment mounted to the arm, a swirl velocity of up to $22.5 \%$ of the rotational speed of the arm was found to occur ${ }^{10,11}$. 
For this new experimental design the same flow conditions can be maintained irrespective of the number of rotations undergone by the wind tunnel model. This allows the condition to be sustained, and offers the potential for detailed investigation and practical development within curved flow (or in the cornering environment).

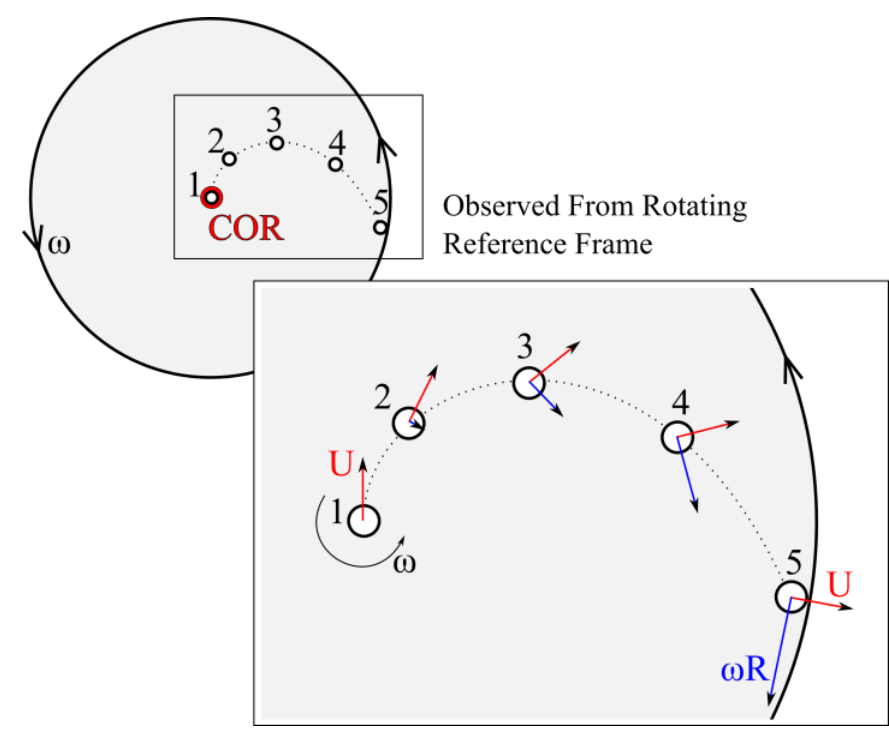

Figure 3. The motion of a fluid particle entering at the centre of a rotating reference frame, with a constant velocity defined in the absolute reference frame

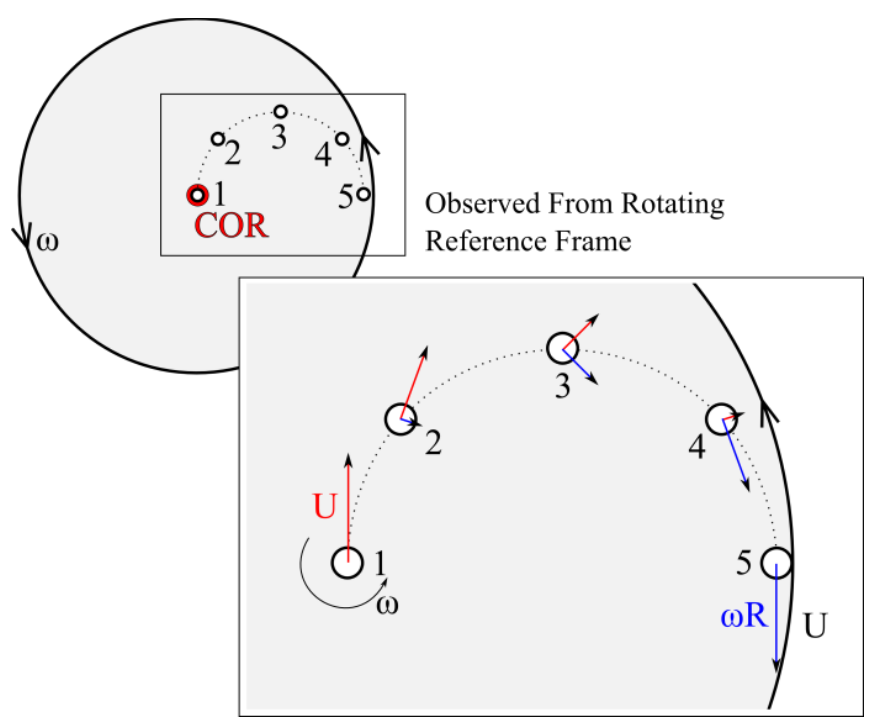

Figure 4. The motion of a fluid particle entering at the centre of a rotating reference frame and maintaining a constant velocity while increasing radial displacement

\section{CFD Analysis}

Reynolds Averaged Navier Stokes (RANS) simulations were used to investigate the design prior to construction of the prototype. The $\mathrm{k}-\omega \mathrm{SST}$ turbulence model ${ }^{12}$ was used with a low-Re wall adaption which was suitable given the considered $\operatorname{Re}^{13}$.

An overview of the boundary conditions of the model is shown in Fig. 5 with a detail view of the hybrid computational grid. The inlet was modelled at the location of the fan with a constant velocity of $2.22 \mathrm{~m} / \mathrm{s}$ which corresponded to a test-section Re of $7.85 \times 10^{5}$, based on test-section height. The inlet and downstream straight 
cylindrical tunnel section were modelled as a separate volume zone with a stationary reference frame and non-slip walls.

Lower tunnel sections were modelled as a rotating reference frame with a constant angular velocity of $6.28 \mathrm{rad} / \mathrm{s}$. Honeycomb screens positioned at both the start and end of the transition were modelled as a zero planar velocity porous regions. All lower internal walls were modelled as non-slip boundaries, while the outlet was specified as a zero static pressure outlet. This outlet simplification did not account for external disturbances created by the tunnel due to its motion through the surrounding air. Initial isolated simulations of the outlet with an external disturbance indicated that the length of the test section ensured external effects would likely only have a small impact on the flow quality, however it is acknowledged that the outlet condition was not accurately representative of reality.

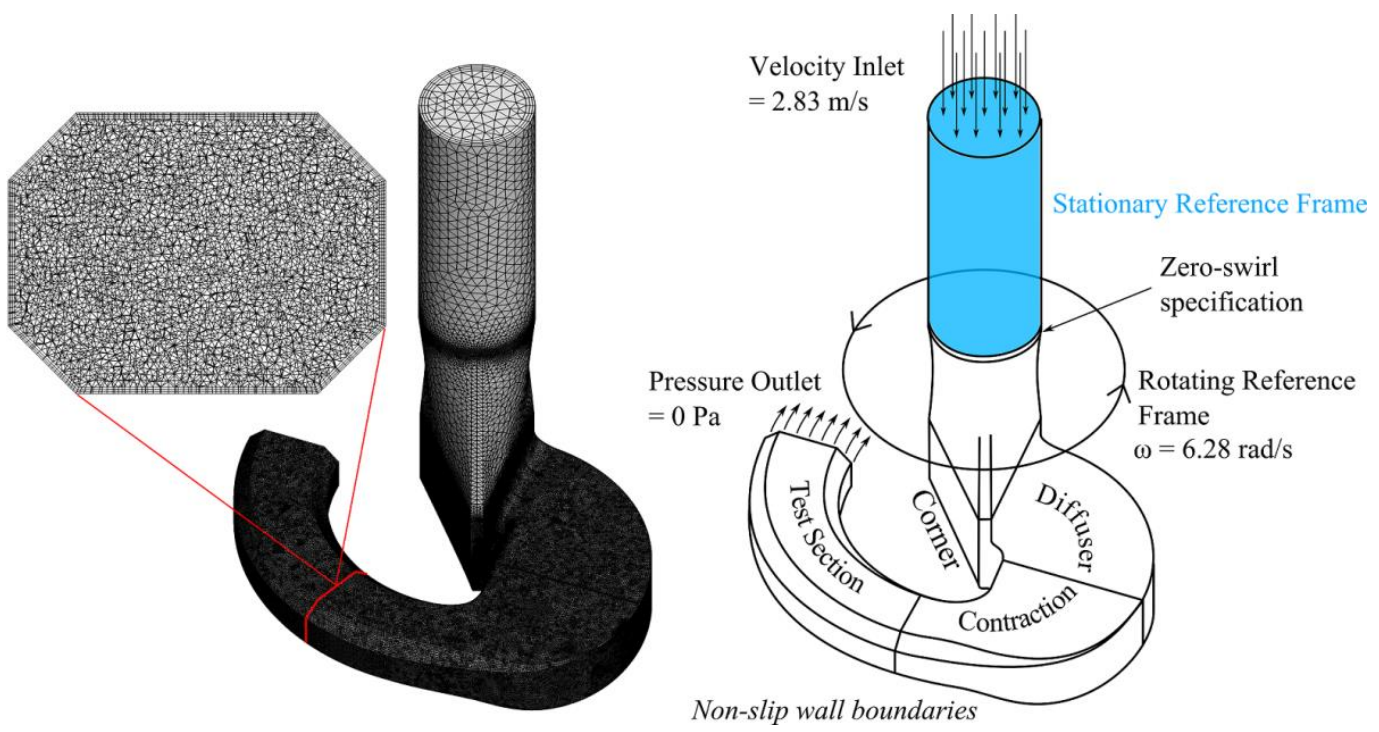

Figure 5. Detail of the grid and overview of the boundary conditions used in the numerical model

A pressure-based implicit coupled solver was utilized to achieve steady-state simulations. Compressibility effects at the simulated Mach numbers were deemed negligible and permitted the assumption of incompressible flow. Simulations were run using a second-order node-based upwinding discretization scheme across 64 processors. Three point velocity monitors were placed downstream of the trailing edge of the centre turning vane, at the start of the contraction nozzle, and at the test-section entrance. Convergence was deemed to be met when velocity values ceased to change by more than $0.005 \%$ over 1000 continued iterations, and this was achieved after 9000 iterations.

The geometric complexity of the corner region due to the presence of the turning vanes, and the necessity of using an efficient meshing procedure that could adapt to design changes, favored the adoption of an unstructured tetrahedral meshing procedure, as is shown in Fig. 5. Cells were focused in the region of the turning vanes, the contraction nozzle, and the test section with these regions identified as most critical to the overall performance. Different grid densities were evaluated at several stages throughout development with mesh sizes ranging from $3.9 \times 10^{6}$ to $3.0 \times 10^{7}$, the final grid adopted consisted of $1.8 \times 10^{7}$ cells and was used for all full tunnel simulation results that are referred to.

\section{Design}

\section{A. Axial Fan}

A means to overcome the system losses and maintain the correct velocity profile in the test section was required, and due to low system losses it was determined that an axial fan was sufficient to provide the required pressure rise. A $450 \mathrm{~mm}$ diameter aerofoil profiled fan was utilized and gave a fan to test-section area ratio of 2.12 . The inlet to the axial fan was smoothed with the use of an elliptically profiled bellmouth with an upstream safety screen.

Hypothetically the entire tunnel could be placed on a turntable, including the axial fan and casing, bellmouth, and the straight tunnel section. The rotation of the experimental rig in its entirety would only result in a minor benefit or detriment to the performance of the fan. However, a practical consideration for the design was to reduce the rotating mass. The fan and the straight tunnel section downstream of the fan, were fixed to a stationary frame positioned above the rotor component. 


\section{B. Rotary Joint}

As the flow passes from the stator section to the rotor, a simple overlapped circular joint with a seal was manufactured to ensure losses were minimized. In order to stabilize the rotor due to imbalances, and ensure the accurate alignment between stator and rotor, a central shaft was positioned between the two parts. The shaft was positioned in a plain bearing connected by four slender arms to the rotor, as is shown in Fig. 7.

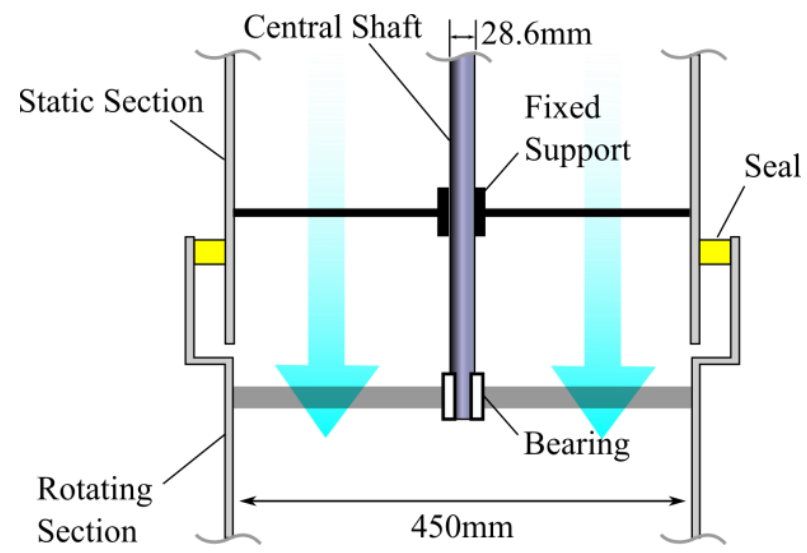

Figure 6. Diagram of the rotary joint and central shaft configuration

\section{Corner}

By passing the flow down through the central axis it enables the positioning of the fan in a location where it can still function as designed. It also ensures that while the flow is travelling in the vertical axis there is minimal influence due to the rotation of the tunnel. Once the flow reaches the plane of the test section it is required to turn through a $90^{\circ}$ bend. A seven vane cascade is used to turn the flow into the plane of the test section. A Gelder ${ }^{14}$ based vane profile was selected, as has been used in a number of established wind tunnels.

\section{Diffuser}

By maintaining a constant cross sectional area while displacement from the centre of rotation increases, the flow is decelerated in the absolute reference frame, and retains constant velocity in the relative reference frame. A $600 \times 250 \mathrm{~mm}$ constant cross-sectional area was maintained with an expansion angle to allow for boundary layer growth to ensure a constant circular arc with a mean radius of $375 \mathrm{~mm}$. While not similar in appearance to a typical diffuser on a conventional wind tunnel, the section performs a similar function.

When in a rotating reference frame the total pressure increases with the distance from the centre of rotation due to an increase in the dynamic pressure according to $q=0.5 \rho(\omega R)^{2}$. Thus as the radius $(R)$ increased, so did the dynamic pressure proportional to its square. Neglecting significant losses it can be observed that if a constant dynamic pressure is maintained while the radius increases an increase in static pressure will occur, and the velocity will decrease within the absolute reference frame, as is shown in Fig. 7.

\section{E. Contraction}

The contraction accelerated the flow to where it is stationary within the absolute reference frame, or equal to $\omega \mathrm{R}$ within the relative reference frame, as shown in Fig. 8. Wall curvature was optimized through the CFD simulation. Initial design iterations were based on an adapted matched cubics profile using a two dimensional shape for manufacturing simplicity. The tunnel height remained a constant $250 \mathrm{~mm}$ from the corner through to the exit of the test section.

Numerical simulation results indicated that there was potential for separation to occur on the inner side of the contraction due to the steep pressure gradient. Smoothing the inside profile to a more gentle curvature aided in maintaining attached flow. Typically a shorter contraction would be desirable for flow quality in the test section ${ }^{15}$, however due to the differing lengths of the two sides of the nozzle, the inner side dictated the overall length. 

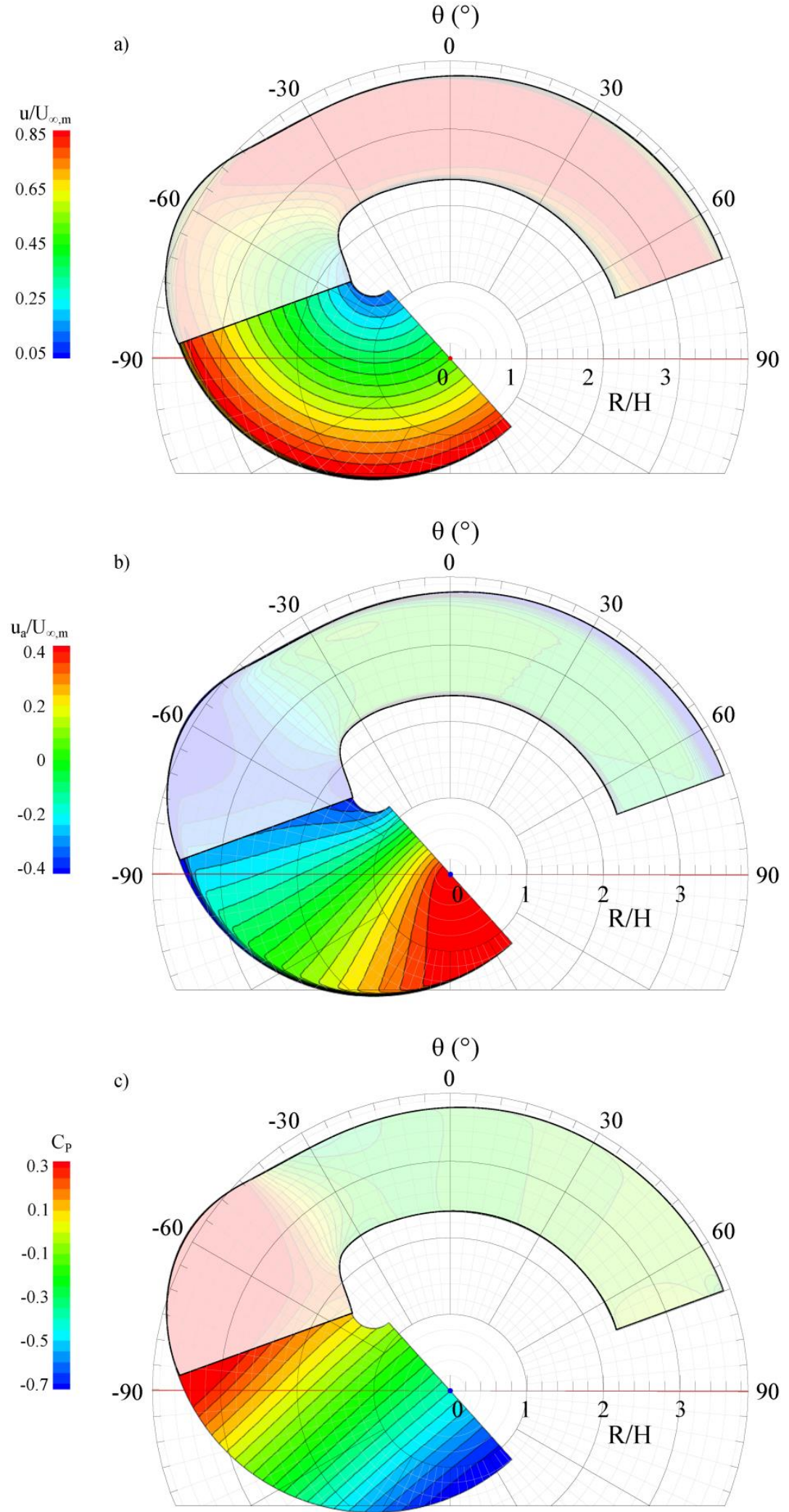

Figure 7. Computational analysis results of diffuser section a) relative velocity distribution, b) absolute velocity distribution, and c) static pressure distribution 

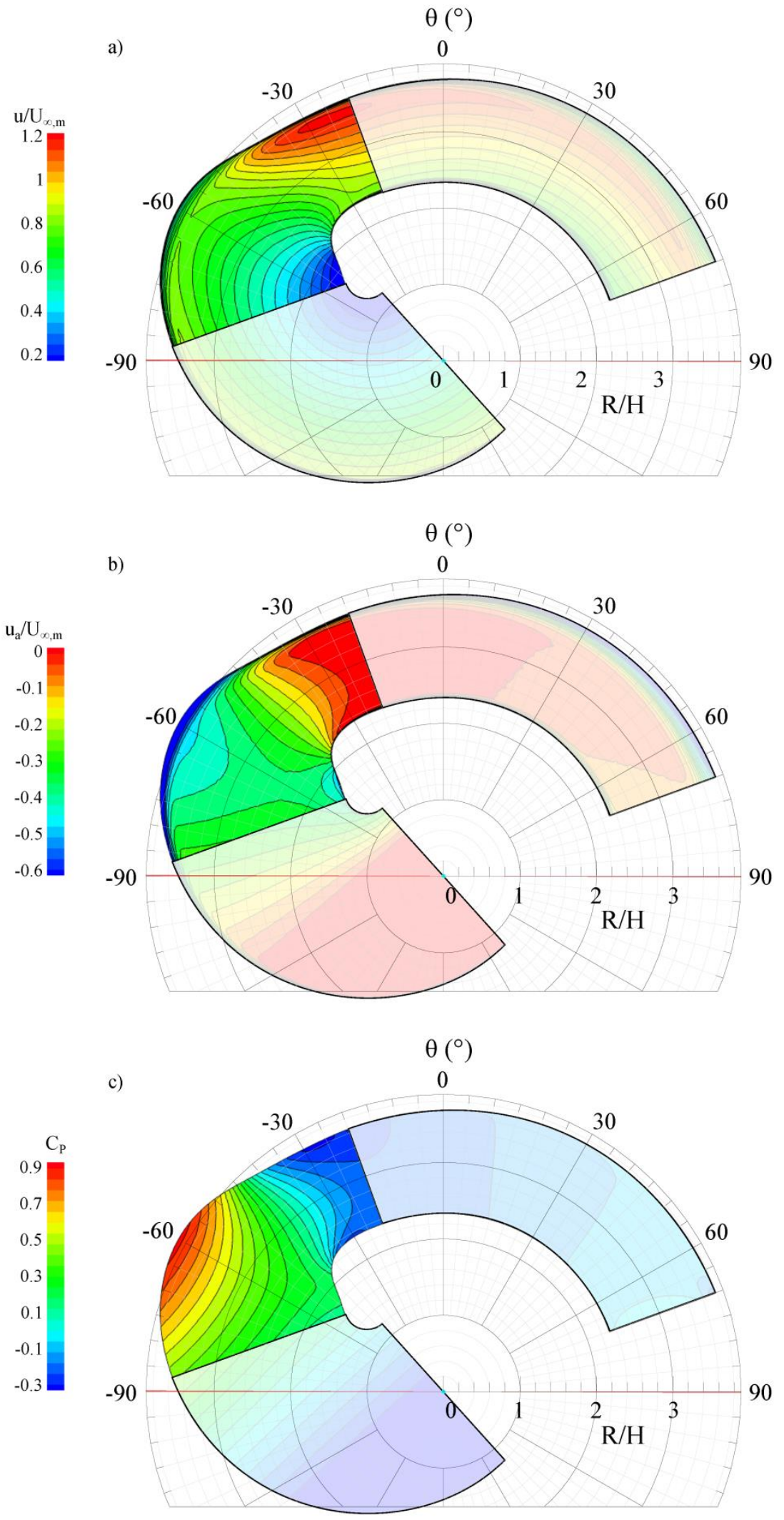

Figure 8. Computational analysis results of contraction section a) relative velocity distribution, b) absolute velocity distribution, and c) static pressure distribution 
Furthermore, to reduce the formation of vortices within the contraction nozzle it was found preferable to develop corner chamfers along the nozzle which resulted in an octagonal profile for the test section. A relatively small contraction ratio of 2:1 was the final design configuration. Higher contraction ratios are typically beneficial toward reducing turbulence intensity ${ }^{15}$, but in this instance presented packaging issues and demonstrated greater propensity for flow separation near the exit on the inner side.

\section{F. Test Section}

The test section had maximum cross-sectional dimensions of $336 \times 225 \mathrm{~mm}$ which gave a cross sectional area of $0.075 \mathrm{~m}^{2}$. Perspex windows were built to permit optical access into the section and allow flow visualization.

For all rotating tunnel sections the change in velocity across the width meant non-uniformity in boundary layer growth on the inner and outer sides. CFD analysis was used to approximate this disparity. Due to this the outer side expands at a $1.1^{\circ}$, while the inner at $0.3^{\circ}$, as is shown in Fig. 9, to allow for this boundary layer growth along the test section walls and ensure flow uniformity along the length of the test section.

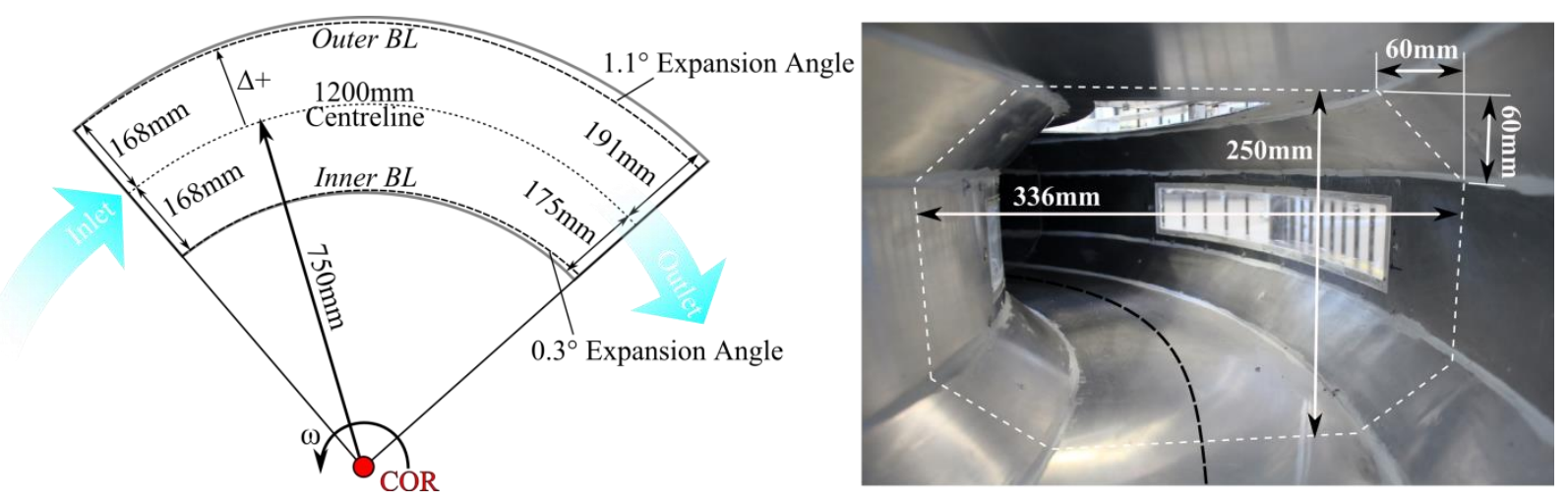

Figure 9. Test section dimensions with expansion angle to accommodate boundary layer growth

\section{G. Outlet}

When correctly calibrated the air in the test section is stationary relative to the surrounding environment (or absolute reference frame). Having the outlet at the end of the test section means the tunnel is essentially filling its own wake, returning stationary air to the room. The motion of the rest of the tunnel through this air then deflects it radially outwards. In reality, the rotor generated an external wake which resulted in the test-section static pressure remaining below ambient and resulted in some flow non-uniformity which is discussed further in Section VII.

\section{Construction}

Minimizing the rotating mass was a key consideration in the design of the experimental rig. All tunnel sections were constructed from sheet aluminum, and the curvature provided additional stiffness to the sections. This allowed thin sheet to be used for a number of large sections.

A three-phase AC motor and gearbox were used to drive the rotor as shown in Fig. 10. Both were attached to a base-plate that was bolted directly to the ground. A bearing housing with a thrust bearing aided in supporting the vertical load. The motor speed was modulated through the use of a variable frequency drive.

The rotor was statically balanced prior to operation to minimize vibration. The entire rotor was aligned horizontally and supported using a central shaft. A balancing arm was positioned directly opposite the determined centre of imbalance and additional mass was then attached to the arm until the rotor component spun freely. This method proved effective and prevented vibration becoming a source of measurement error, or causing mechanical damage.

\section{Instrumentation}

The rotation of the test section introduced additional considerations when selecting suitable instrumentation to investigate the flow. The maximum design speed resulted in centripetal acceleration up to $163.1 \mathrm{~m} / \mathrm{s}^{2}$, however the maximum acceleration reached during the present experimental program was limited to $56.6 \mathrm{~m} / \mathrm{s}^{2}$. It was therefore necessary to ensure all equipment was operable under this acceleration. 


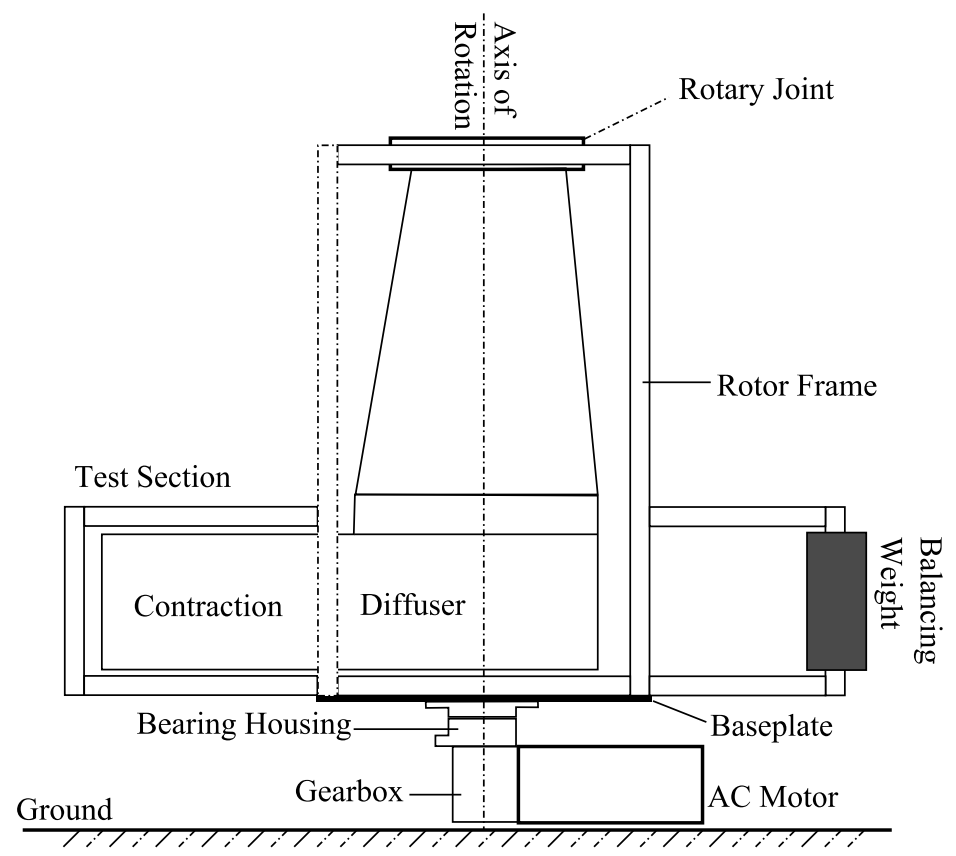

Figure 10. The direct drive system used to spin the rotor component of the tunnel

A lightweight manual traversing system was used which secured in two curved rails which were external to the test section. The traverse used a vertical support strut which could be manually adjusted within an extruded channel to position probes as required. Accuracy was estimated based on physical measurement of probe position compared to indicated position. Vertical location (z) was accurate to $\pm 2 \mathrm{~mm}$, radial position (R) to $\pm 3 \mathrm{~mm}$, and angular position along the test section $(\theta)$ was accurate to within $\pm 0.15^{\circ}$. The locations of horizontal profile measurements taken within the test-section are shown in Fig. 11.

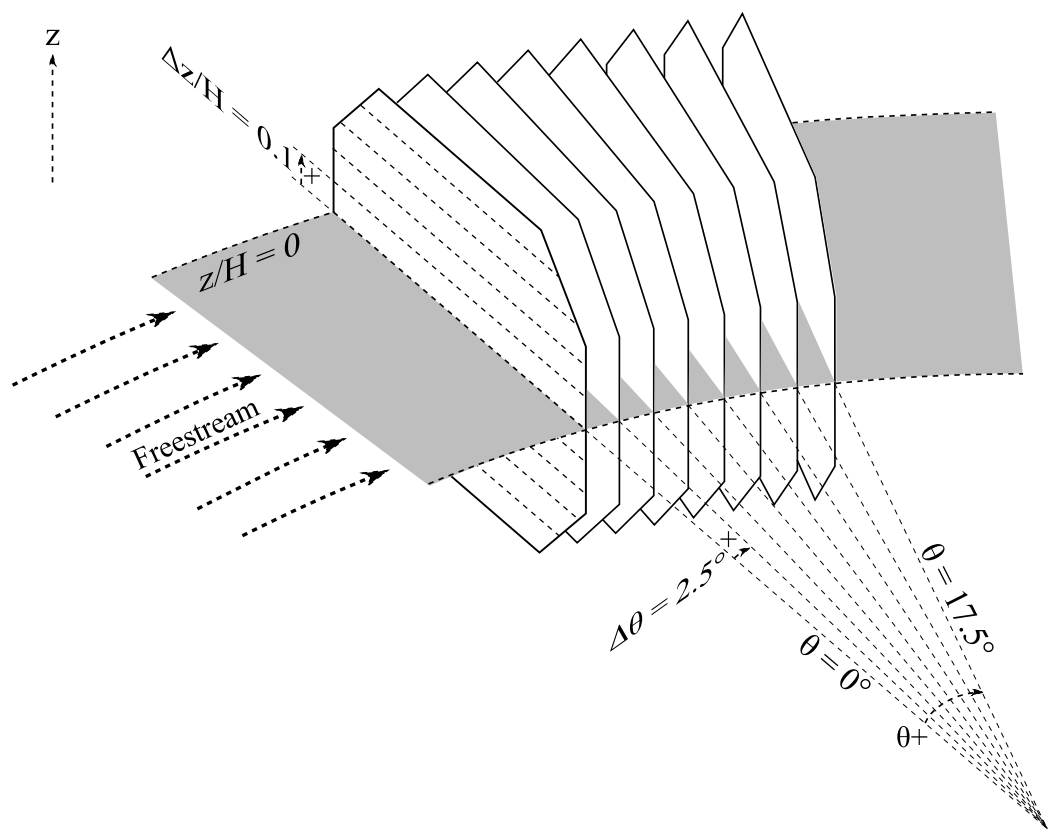

Figure 11. Location of velocity and static pressure measurements taken throughout the test-section 
A rotary incremental encoder (British Encoder 755/HV - 068647) with 720 pulses per rotation was attached directly to the motor to monitor the angular velocity. As the gearbox had a 10:1 reduction ratio the encoder produced 7200 pulses per single rotation of the rotor section of the tunnel. Readings were recorded as averaged values over a period of $0.1 \mathrm{~s}$ and angular velocity was remained within $\pm 0.02 \mathrm{rad} / \mathrm{s}$ of the reported value for all experiments.

A Dantec Dynamics Multichannel CTA system (54N82) was used for flow velocity and turbulence intensity measurements. The system was secured to the rotor section of the tunnel and measurements were taken by means of a four-probe rake mounted to the traverse, as shown in Fig. 12. A four-channel 24bit signal acquisition module (NI 9234) was used to record voltage outputs, and this was positioned in a National Instruments Wireless carrier (NI WLS-9163) which sent data via a Wi-Fi connection to an external PC. All equipment was mounted externally above the test-section as shown in Fig. 13. Data was averaged over a 10 s sampling period at $12.8 \mathrm{kHz}$ producing $1.28 \times 10^{5}$ samples with each measurement.

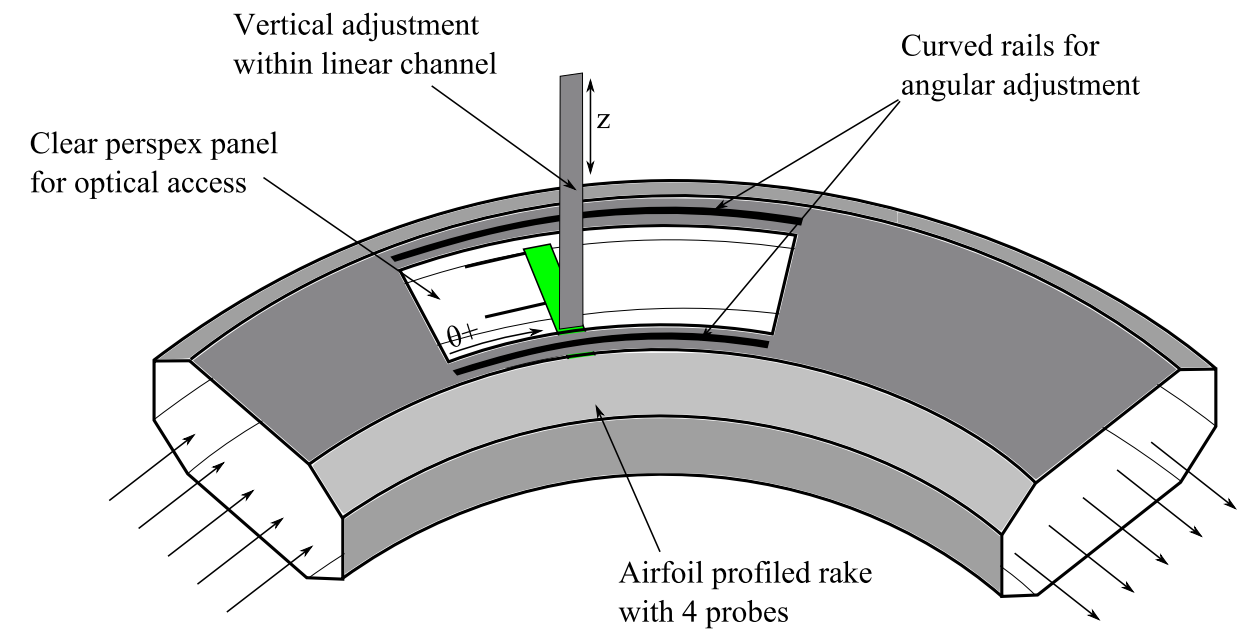

Figure 12. Diagram of pitot rake and static rake positioned within the test section
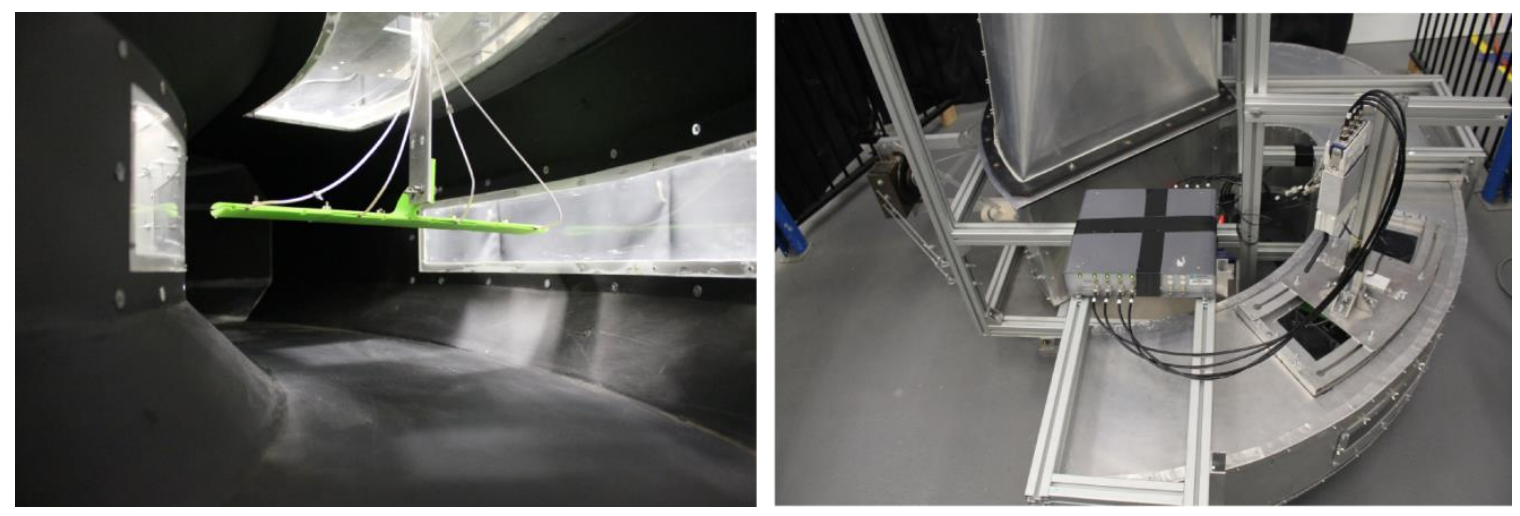

Figure 13. Instrumentation positioned externally and internally on the test-section

Static pressure measurements were captured through a static rake consisting of four ports connected to digital low differential pressure LDE transducer by First Sensor AG. The pressure sensors were mounted externally to the test section. A four-channel 24bit signal acquisition module (NI 9219) was used to record voltage outputs, also positioned in a National Instruments Wireless carrier. Differential pressure measurements between a reference value and the test section were taken at a frequency of $100 \mathrm{~Hz}$. All measurements were averaged over a period of $10 \mathrm{~s}$ with a correction applied to accommodate acceleration within the rotating reference frame.

A TSI incorporated automatic calibrator was used with an MKS Instruments Baratron Manometer (220DD) to calibrate the hot-wire probes and pressure sensors. For hot-wires a 40 point calibration from 2.5 to $35 \mathrm{~m} / \mathrm{s}$ was conducted using a $\mathrm{x}^{10}$ distribution which weighted calibration points toward lower velocities. A King's law curve 
fit $^{16}$ was identified as superior to a polynomial fit within the low velocity range and was favored in this instance. The maximum absolute calibration error was $0.067 \mathrm{~m} / \mathrm{s}$ for all probes within the considered velocity range.

A 40 point distribution of pressures up to $100 \mathrm{~Pa}$ with an $\mathrm{x}^{10}$ distribution. An ordinary linear least-squares fit was used to determine the relation between voltage and pressure, with an initial zero-offset value. The maximum absolute error was $0.015 \mathrm{~Pa}$ within the recorded range of pressures.

All results were repeated three times with the mean value shown. Due to the number of samples recorded at each location not being sufficient to confidently conduct a statistical interpretation of the error, an estimate was calculated based on an Nth-order uncertainty analysis described by Moffat ${ }^{17}$ for a single sample measurement data set. A corresponding $95 \%$ confidence interval is indicated accordingly on each figure.

\section{Results}

\section{A. Flow Visualization}

Smoke flow visualization was used as a qualitative technique to confirm the curved path of the flow within the test section. This was achieved through the use of incense positioned along a horizontal rake with the leading edge at $\theta=5^{\circ}, \mathrm{z} / \mathrm{H}=0$. The positioning on the incense resulted in split longitudinal wake structure which demonstrated a tendency to diverge. This was a mild hindrance, however the position was favorable over adopting a vertical alignment which resulted in a Von Kármán street type mechanism. The temperature difference also resulted in vertical buoyancy which caused the smoke streams to rise in the vertical axis within the test-section.

The method proved effective at low velocity and the image shown in Fig. 14 was conducted at $1.57 \mathrm{rad} / \mathrm{s}$ which corresponded to $\mathrm{U}_{\infty, \mathrm{m}}=1.18 \mathrm{~m} / \mathrm{s}$. The $\Delta / \mathrm{H}$ is a dimension introduced as equivalent to radial displacement from the centerline of the test-section divided by test-section height.

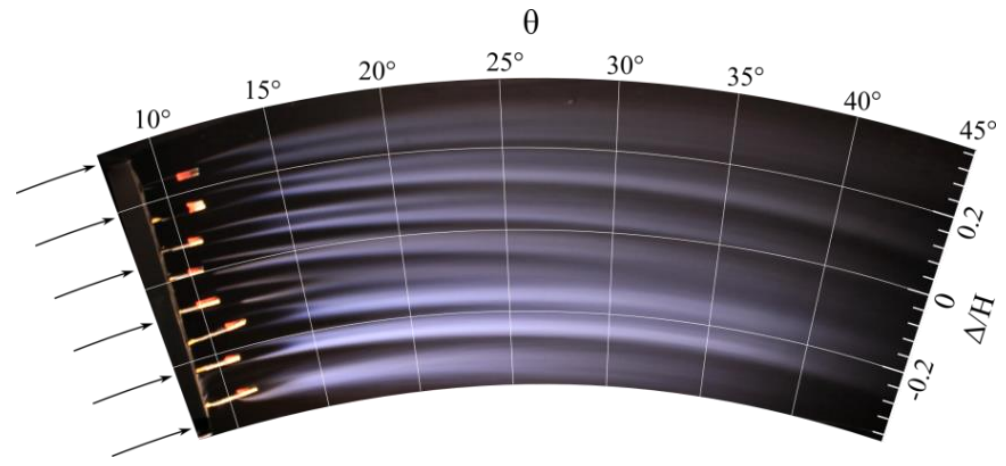

Figure 14. Smoke flow visualization conducted at $U_{\infty, m}=1.18 \mathrm{~m} / \mathrm{s}$

This visualization confirmed the flow to assume a curved path along the length of the window considered. Furthermore this demonstrated alignment with the geometric shape of the tunnel section. While this identified the expected curved flow within the test-section, further quantitative assessment of flow properties was necessary to determine successful operation.

\section{B. Flow Measurement}

Different mean freestream velocities with the correct corresponding angular velocities were measured ranging between $\mathrm{U}_{\infty, \mathrm{m}}=3.53 \mathrm{~m} / \mathrm{s}$ to $5.89 \mathrm{~m} / \mathrm{s}$. Results were taken at seven point locations across the width of the test-section allowing the extraction of horizontal flow profiles. These profiles were taken at nine vertical locations, as shown in Fig. 11, resulting in a distribution of measurements throughout the test-section. A representative horizontal profile taken across the span of the test-section is shown in Fig. 15a) indicating velocity, static pressure and turbulence intensity for a condition where the freestream flow speed and angular velocity are correctly balanced according to $\mathrm{U}_{\infty}=\omega \mathrm{R}$, as was designed.

Velocity measurements correlated with the theoretically ideal distribution and demonstrated an increase in the relative tangential velocity as shown in Fig. 15a) and b). Similar flow consistency was identified to occur throughout the investigated planes and a mean coefficient of determination was used to assess the overall fit of the velocity distribution. Across all measurements $\mathrm{R}_{\text {mean }}^{2}=0.901$ for $\mathrm{U}_{\infty, \mathrm{m}}=3.53 \mathrm{~m} / \mathrm{s}, \mathrm{R}_{\text {mean }}^{2}=0.914$ for $\mathrm{U}_{\infty, \mathrm{m}}=4.71 \mathrm{~m} / \mathrm{s}$, and 
$\mathrm{R}_{\text {mean }}^{2}=0.928$ for $\mathrm{U}_{\infty, \mathrm{m}}=5.89 \mathrm{~m} / \mathrm{s}$, which indicated improved overall correlation between the theoretical and experimental velocity distribution as Re increased. A consistent discrepancy throughout all results was a relative decrease which occurred between $\Delta / H=0.36$ and 0.54 . Between these locations an increase in freestream velocity would be expected, however measurements indicated a reduction. Across all measurements the maximum difference between $\Delta / \mathrm{H}=0.36$ and 0.54 was $-0.026 \mathrm{u} / \mathrm{U}_{\infty, \mathrm{m}}$ at $\mathrm{z} / \mathrm{H}=0$ for $\theta=0^{\circ}$, which opposed the expected theoretical increase of $0.06 \mathrm{u} / \mathrm{U}_{\infty, \mathrm{m}}$

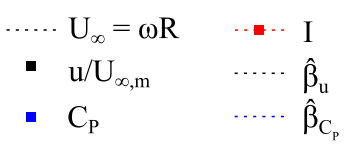

$$
\begin{aligned}
\mathrm{m}_{\mathrm{C}_{\mathrm{p}}}(\text { ideal }) & =0.0 \underset{\Delta / \mathrm{H}}{\mathrm{C}_{\mathrm{P}}} \\
\mathrm{m}_{\mathrm{u}}(\text { ideal }) & =0.333 \underset{\Delta / \mathrm{H}}{\mathrm{U}_{\infty, \mathrm{m}}}
\end{aligned}
$$
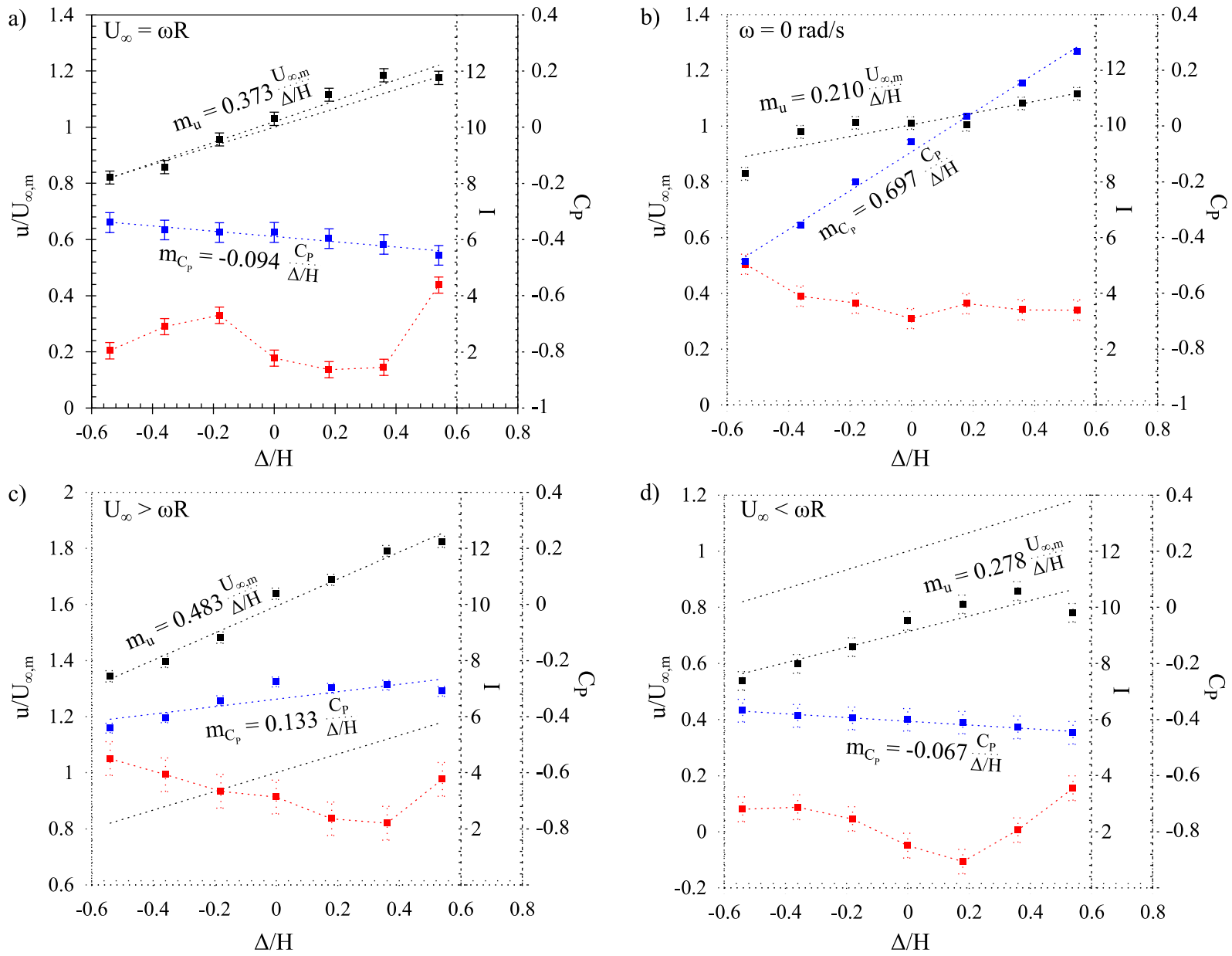

Figure 15. Velocity, static pressure, and turbulence intensity measurements within the test-section at a) $\left.\left.U_{\infty, m}=3.53 \mathrm{~m} / \mathrm{s} \mathrm{b}\right) U_{\infty, m}=4.71 \mathrm{~m} / \mathrm{s} \mathrm{c}\right) U_{\infty, m}=5.89 \mathrm{~m} / \mathrm{s}$

The distribution of static pressure throughout the test-section remained near-constant, however a consistent trend was observed to occur in the radial direction. In Fig. 15a) a negative gradient of $-0.094 \Delta \mathrm{C}_{\mathrm{P}} / \mathrm{H}$ can be observed to occur when the ideal condition should exhibit constant static pressure, however this gradient was observed to reduce as measurements were recorded further downstream. This effect was attributed to the design of the contraction wall curvature which resulted in a locally accelerated region and static pressure decrease near the exit on the outer side, which continued into the test-section and resulted in this measured non-uniformity, highlighting a region for future development.

The design did not utilize any turbulence or flow-straightening screens to allow improved understanding of the flow, and due to the small contraction ratio, the turbulence intensity remained high with an average intensity of $2.61 \%$ recorded throughout the test section. The maximum recorded value was $5.25 \%$ toward the lower surface of 
the test-section, with a minimum recorded value of $1.10 \%$. Consistently the highest turbulence intensities were measured within $0.1 \mathrm{H}$ of the lower test-section surface with this being the only location where local values exceeded $5 \%$, and this was primarily attributed to the two-dimensional shape of the contraction. A similar but milder increase also occurred towards the upper surface, further confirming this. The lowest turbulence intensities were concentrated centrally and slightly toward the outer side, with minimum values recorded at $\Delta / \mathrm{H}=0$ and 0.18 between $\mathrm{z} / \mathrm{H}=0.1$ and -0.1. The inconsistency of the turbulence intensity was attributed to a range of effects which occurred over the turning vanes, with this being a particularly complex region of the design.

\section{Angular and Freestream Velocity Ratio}

The rotation of the lower tunnel sections presented the most significant challenge from a mechanical design perspective, and inherently makes the present configuration more difficult to scale than conventional designs. When operating correctly only one freestream velocity is suitable for any given angular velocity. This is due to the relation between these parameters being defined according to $U_{\infty}=\omega R$ where $R$ values remain fixed. The effect of having an imbalanced relationship between these parameters was also analyzed to gain further understanding of the function.

In Fig. $15 b$ ) the mean freestream velocity remained at $U_{\infty, m}=4.71 \mathrm{~m} / \mathrm{s}$ while the rotor was stationary, and this caused a loss in the uniformity of the velocity gradient across the test-section. The magnitude of the total difference between $\Delta / \mathrm{H}=-0.54$ and $\Delta / \mathrm{H}=0.54$ was $79.7 \%$ that of the theoretically ideal flowfield, however between $\Delta / \mathrm{H}=-$ 0.36 and $\Delta / \mathrm{H}=0.18$ the profile remained more constant with a maximum change of only $0.033 \mathrm{U}_{\infty}$ across the four measurement locations, resulting in a reduced gradient for the linear fit across the seven recorded points.

To balance the induced flow rotation within the test-section, a significant static pressure gradient occurred. This resulted in a minimum static pressure occurring on the inner side, and maximum on the outer, where the difference between $\Delta / \mathrm{H}=-0.54$ and 0.54 was a pressure coefficient of 0.753 . Thus the change in static pressure significantly exceeded that of dynamic pressure, and resulted in the occurrence of suction and pressure surfaces on the inner and outer wall respectively.

The mean turbulence intensity was $3.74 \%$, which was higher than the calibrated condition shown in Fig. 15a), however the intensity was more consistent throughout the test section, and was attributed to increased mixing of the flow.

Figure 15c) and d) show representative profiles for conditions where the freestream velocity is higher and lower than the designed value with respect to the angular velocity. The conditions were respectively achieved through increasing the fan speed, and turning the fan off. Increased freestream velocity resulted in a more significant gradient across the test-section $\left(0.483 \mathrm{U}_{\infty, \mathrm{m}} / \mathrm{H}\right.$ as compared to $0.373 \mathrm{U}_{\infty, \mathrm{m}} / \mathrm{H}$ for the calibrated condition), the profile also demonstrated reduced linearity across the width. The reduced flow velocity resulted in a comparatively more slight gradient of $0.278 \mathrm{U}_{\infty, \mathrm{m}} / \mathrm{H}$ and a consistent linear increase with the exception of the measurement at $\Delta / \mathrm{H}=0.54$, where a decline of $0.077 \mathrm{U}_{\infty, \mathrm{m}}$ occurred as opposed to the expected theoretical $0.060 \mathrm{U}_{\infty, \mathrm{m}}$ increase, which continued to be a common discrepancy across most analyzed conditions.

In Fig. 15c) a rise in static pressure occurred from $\Delta / \mathrm{H}=-0.54$ to 0 , but levelled to remain relatively constant on the outer side. The net result was a mildly increasing mean gradient of $0.133 \Delta \mathrm{C}_{\mathrm{P}} / \mathrm{H}$ from the inner to outer side. This was attributed to two separate, opposing effects. The increase in freestream velocity with respect to angular velocity results in curvature being introduced to the flow with the associated static pressure gradient, albeit to a more minor extent than occurred in Fig. 15b). However, the low pressure region created on the outer wall of the contraction profile partially mitigated this effect on the outer side. The same discontinuity in the static pressure profile can be observed in both Fig. 15b) and c) with a central kink in the profile.

For the increased freestream condition the test-section geometry enforces an absolute reference frame curvature on the flow in the direction of the test section curvature itself, and thus results in the outer side acting as a pressure surface and the inner becoming a suction surface. When the freestream flow velocity is less than $\omega \mathrm{R}$, the enforced curvature within the absolute reference frame acts in the opposite direction, where the inner surface acts as a pressure surface while the outer becomes a suction surface. Due to this effect it was expected that the reduced freestream condition shown in Fig. 15d) would result in a mildly more positive static pressure gradient with respect to the calibrated condition, and was confirmed by the results. This positive gradient reduced opposed the negative gradient induced by the contraction to result in a milder, but still negative, gradient of $-0.067 \Delta \mathrm{C}_{\mathrm{P}} / \mathrm{H}$.

For the conditions shown in Fig. 15c) and d), turbulence intensity was calculated with respect to the measured velocity profile as opposed to the $\omega \mathrm{R}$ value. This was to ensure that increased or decreased freestream velocity did not produce a misrepresentative change in turbulence intensity. The increased freestream velocity, shown in Fig. 15c) resulted in an increase in the mean turbulence intensity to a value of $3.28 \%$. For the reduced freestream condition the intensity decreased to a mean value of $2.29 \%$. In both cases increased uniformity was identified to occur across the width of the section which was attributed to increased mixing. 


\section{Discussion}

\section{A. Limitations}

Results demonstrate that the concept is capable of producing continuous flow conditions that are representative of those experienced by an object as it assumes a curved path through stationary flow, however the present design also has a number of limitations, which are dependent on the intended use.

In comparison to a traditional wind tunnel the rotating component would make it more difficult to scale due to the additional mechanical requirements. As the air within the test-section is stationary relative to the surrounding reference frame it must be considered that the desired relative freestream velocity becomes equivalent to the tangential velocity of the test-section itself. In simple terms to achieve $30 \mathrm{~m} / \mathrm{s}$, the test-section needs to travel at $30 \mathrm{~m} / \mathrm{s}$.

The proof-of-concept design is limited to testing only one corner radius. It would be possible to test different relative corner radii by using different scale models, and then conducting analysis at a common Re by using different flow speeds, as is shown in Fig. 16. The simplest method to allow multiple corners using the one model would be to use a high aspect ratio test-section, with enough width for the model to be swept through different radii. Another, likely more complex, solution would be the use of flexible walls for the sections. A change in curvature radii would need to accommodate for the test-section being positioned further from the centre of rotation, so the diffuser and contraction would also need to be flexible, and increase or decrease in length as required.

a)

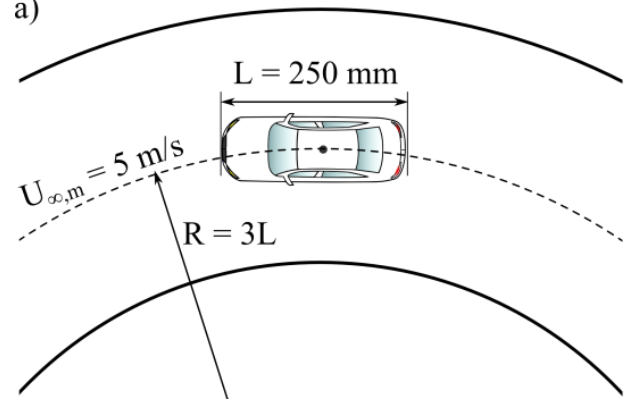

b)

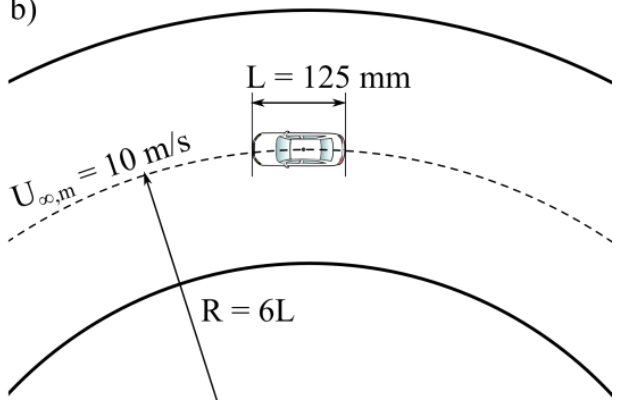

Figure 16. Illustration of how different effective corner radii could be achieved within a fixed curvature testsection at the same Re showing a a) $3 \mathrm{~L}$ radius corner, and b) $6 \mathrm{~L}$ radius corner

The inconsistent turbulence intensity and non-uniformity were undesirable for immediate experimental analysis of any model. The inclusion of turbulence screens would be an effective method to improve these current shortcomings. Screens would need to be non-uniform to ensure a uniform static pressure drop within the nonuniform velocity field. Barlow et. $\mathrm{al}^{15}$ gave guidelines on calculating the pressure drop across screens, and this can be modified to calculate the appropriate wire width and change in cell size required for a given velocity gradient.

It must be noted that the omission of any screens in this initial proof-of-concept phase was deliberate to ensure that any effects due to tunnel features were not masked by screens, and the velocity field was achieved without requiring the correction provided by non-uniform screens, as has been used previously in a stationary wind tunnel section $^{18,19}$.

\section{B. Application Notes}

For automotive-specific testing a moving ground can be an important feature. This will typically be achieved by using a belt positioned underneath the model that moves at the same speed as the freestream flow. For the present design the air within the test-section is stationary within the absolute reference frame, so the moving ground would need to be a stationary platform rather than a moving belt. This is not a feature present on the current design but is something that could be accommodated for in a future version.

An important further note is that the experimental rig is not required to be entirely built as its own dedicated facility. Provided there is sufficient room to accommodate the desired rotor size, the design could be potentially be incorporated into an existing wind tunnel circuit. Dependent on a variety of circumstantial factors, this may be achieved most easily as an additional branch off the main circuit or an in-line configuration. As with all wind tunnels, the experiment simply requires a pressure rise that is sufficient to overcome the system losses, and the most cost-effective way to achieve this may be through utilization of existing resources. 


\section{Conclusion}

A new method developed for creating continuous and repeatable curved flow has been proposed and investigated through the construction and experimental analysis of the proof-of-concept for a new type of wind tunnel. Results demonstrated the experimental concept was capable of achieving flow conditions that were closely representative of the theoretical flow properties for a $750 \mathrm{~mm}$ mean radius corner. The design uniquely achieved these conditions in the absence of a significant static pressure gradient, or using non-uniform screens to correct the freestream velocity distribution.

Results demonstrate that the design was capable of maintaining the flow conditions consistently throughout the test-section in the vertical and downstream directions. These results were able to be achieved across a range of mean freestream velocities from $3.53 \mathrm{~m} / \mathrm{s}$ to $5.89 \mathrm{~m} / \mathrm{s}$. The experiment established the requirement of an equivalent relationship between the freestream velocity, the angular velocity, and radius toward achieving the correct flow conditions. This demonstrated that by using a rotational, non-inertial reference frame, deflection of the flow due to the Coriolis effect allowed flow curvature to be achieved in the absence of acceleration acting on the fluid. This was then able to uniquely create repeatable freestream conditions following a circular, curved path.

To the knowledge of the authors, this is the first experimental rig to be capable to maintaining correct and repeatable curved flow conditions in close ground proximity. In applications such as automotive aerodynamics this design could permit the first experimental analyses into the unique, and sometimes critical, flow conditions experienced by a vehicle when travelling through a corner.

\section{References}

Toet, W., "Aerodynamics and aerodynamic research in Formula 1," Aeronaut. J. 2013; 117(1187):1-26.

2 Keogh, J., Barber, T., Diasinos, S., and Doig, G., "Techniques for Aerodynamic Analysis of Vehicles Travelling through a Corner," SAE Paper No. 2015-01-0022

3 Keogh, J., Doig, G., Diasinos, S., and Barber, T., "The influence of cornering on the vortical wake structures of an inverted wing." Proceedings of the Institution of Mechanical Engineers, Part D: Journal of Automobile Engineering (2015). doi: 0954407015571673.

4 Keogh, J., Doig, G., Diasinos, S., and Barber, T., "Detached Eddy Simulation of the Cornering Aerodynamics of the Ahmed Reference Model" FISITA World Automotive Conference, Maastricht, the Netherlands, June 2014

5 Tsubokura, M., Ikawa, Y., Nakashima, T., Okada, Y., Kamioka, T. and Nouzawa, T., "Unsteady Vehicle Aerodynamics during a Dynamic Steering Action: 2nd Report, Numerical Analysis,” SAE Int. J. Passeng. Cars - Mech. Syst. 2012; 5(1). doi: 104271/2012-01-0448.

6 Okada, Y., Nouzawa, T., Okamoto, S., Fujita, T. et. al "Unsteady Vehicle Aerodynamics during a Dynamic Steering Action: 1st Report, On-Road Analysis" SAE Paper 2012-01-0446 doi:10.4271/2012-01-0446

7 Nara, K., Tsubokura, M., Ikeda, J., Fasel, U., et. al "Numerical Analysis of Unsteady Aerodynamics of Formula Car during Dynamic Cornering Motion," 32nd AIAA Applied Aerodynamics Conference, June 2014, Atlanta, GA, USA

8 Coriolis, G. G., "Memoire sur les equations du mouvement relatif des systemes de corps," J. Ec. Polytech., 15, 142-154, 1835.

9 Baals, D. and Corliss, W., Wind Tunnels of NASA, NASA accessed October 30, 2014. http://www.hq.nasa.gov/pao/History/SP-440/contents.htm

10 Llewelyn-Davies, M. "The Redesign of the College of Aeronautics Whirling Arm Facility", College of Aeronautics Report No. 8702, 1987

11 Kumar, P. "The College of Aeronautics Whirling Arm Initial Development Tests," CoA Note Aero. No. 174, 1967

12 Menter, F. R., "Two-equation eddy-viscosity turbulence models for engineering applications," AIAA Journal, 1994, 32(8):1598-1605, August. doi: 10.2514/3.12149.

13 ANSYS. ANSYS Fluent 14.5 User Guide, 2015.

14 Gelder, Thomas F., R. D. Moore, J. M. Sanz, and E. R. McFarland. "Wind tunnel turning vanes of modern design." 24th Aerospace Sciences Meeting, January 1986, Reno, Nevada, USA

15 Barlow, J. B., Rae, W. H., and Pope, A., Low-Speed Wind Tunnel Testing, 3rd ed., John Wiley \& Sons, New York, 1999

16 King, L. V. "On the convection of heat from small cylinders in a stream of fluid," Philosophical Transactions of the Royal Society, 1914, 214:373-432.

17 Moffat R J. "Describing the uncertainties in experimental results," Experimental Thermal and Fluid Science, 1988, 1(1):317 doi: 10.1016/0894-1777(88)90043-X.

18 Chambers, J. R., Grafton, S. B., and Lutze, F. H., "Curved Flow, Rolling Flow, and Oscillatory Pure-Yawing Wind-Tunnel Test Methods for Determination of Dynamic Stability Derivatives”. NATO AGARD Lecture Series 1981 No. 114 Dynamic Stability Parameters

19 Bird, J. D., Jaquet, B. M., and Field, L., "Effect of Fuselage and Tail Surface on Low-Speed Yawing Characteristics of a Swept-Wing Model as Determined in Curved-Flow Test-Section of Langley Stability Tunnel,” Technical Report 2483, 1951. 